\title{
Ellipsomicroscopy for surface imaging: A novel tool to investigate surface dynamics
}

Cite as: Journal of Vacuum Science \& Technology A 16, 1117 (1998); https://doi.org/10.1116/1.581243

Submitted: 09 October 1997 . Accepted: 05 January 1998. Published Online: 27 October 1998

G. Haas, T. D. Pletcher, G. Bonilla, T. A. Jachimowski, H. H. Rotermund, and J. Lauterbach

\section{ARTICLES YOU MAY BE INTERESTED IN}

Chemical patterning of Ag(111): Spatially confined oxide formation induced by electron beam irradiation

Applied Physics Letters 93, 233117 (2008); https://doi.org/10.1063/1.3040685

Kinetic oscillations in the catalytic CO oxidation on Pt(100): Experiments

The Journal of Chemical Physics 84, 3519 (1986); https://doi.org/10.1063/1.450238

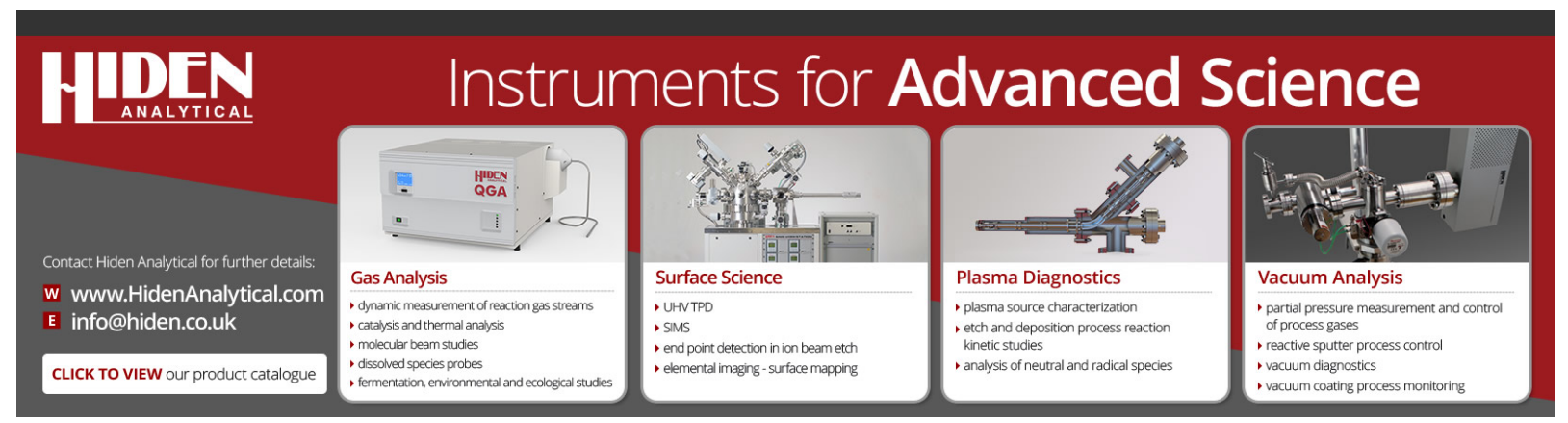




\title{
Ellipsomicroscopy for surface imaging: A novel tool to investigate surface dynamics
}

\author{
G. Haas, T. D. Pletcher, G. Bonilla, and T. A. Jachimowski \\ School of Chemical Engineering, Purdue University, West Lafayette, Indiana 47907-1283 \\ H. H. Rotermund \\ Fritz-Haber-Institut der Max-Planck-Gesellschaft, Faradayweg 4-6, 14195, Berlin, Germany \\ J. Lauterbach ${ }^{\text {a) }}$ \\ School of Chemical Engineering, Purdue University, West Lafayette, Indiana 47907-1283
}

(Received 9 October 1997; accepted 5 January 1998)

\begin{abstract}
This article focuses on the technical issues of imaging of dynamic adsorbate pattern formation on platinum surfaces using laser light to illuminate the area of interest. In particular, ellipsomicroscopy for surface imaging (EMSI) is a powerful tool to follow spatio-temporal patterns of adsorbate layers on catalyst surfaces at arbitrary pressures. This novel method is uniquely sensitive to submonolayer coverages of adsorbates. It expands the range of observable pressure conditions by many orders of magnitude, thus bridging the pressure gap in imaging surface reactions. EMSI is a versatile technique that opens new avenues of potential applications to resolve dynamic surface processes, such as adsorbate diffusion or coating formation. (C) 1998 American Vacuum Society.

[S0734-2101(98)02803-6]
\end{abstract}

\section{INTRODUCTION}

Most experimental techniques used in surface science studies are electron or particle based and are therefore only capable of investigating the surface under ultrahigh vacuum (UHV) conditions. For investigations concerning industrial applications, however, in situ techniques applicable at atmospheric pressures become more important. The requirements for these methods are twofold. First, they must have surface sensitivity, and second, they must be independent from the surrounding pressure. By comparison with other available techniques, optical methods are the best choice. The most common are: ellipsometry, reflection anisotropy spectroscopy (RAS), surface second harmonic generation (SSHG), surface enhanced Raman spectroscopy (SERS), and IR reflection absorption spectroscopy (IRAS). Two of these methods, ellipsometry and RAS, have recently been developed into imaging methods to follow dynamic surface processes: ellipsomicroscopy for surface imaging (EMSI) and reflection anisotropy microscopy (RAM). ${ }^{1,2}$ This article will focus on the technical aspects of the EMSI technique and will show examples of its application to the study of nonlinear phenomena in heterogeneous catalysis.

The initial driving force for the development of EMSI and RAM was the desire to study dynamic patterns on catalyst surfaces at elevated pressures. One particularly remarkable aspect of heterogeneously catalyzed reactions is their ability to oscillate with time, which was first encountered with the $\mathrm{CO}$ oxidation at atmospheric pressures on platinum metals. ${ }^{3}$ This study was followed by the discovery of an abundance of oscillating reaction systems, involving numerous different reactants and types of catalysts (such as polycrystalline wires, meshes, foils, single crystals, and supported

${ }^{a)}$ Electronic mail: jochen@ecn.purdue.edu catalysts). ${ }^{4,5}$ Earlier investigations, however, were limited to studying the temporal behavior of a global quantity such as the overall reaction rate. The nature of oscillations during surface reactions was closely investigated at pressures below $10^{-3}$ mbar using a surface science approach with single crystals under UHV conditions. ${ }^{6-12}$ This investigation led to the explanation of this phenomenon by a LangmuirHinshelwood mechanism in combination with an adsorbate induced change of the surface properties (e.g., by reconstruction or oxidation).

At the gas-solid interface, spatio-temporal patterns are formed by adsorbates and, hence, adsorbate coverages range between zero and one monolayer. ${ }^{13-15}$ Therefore, surface imaging techniques must be sensitive to coverages as small as several hundredths of a monolayer and, at the same time, have appropriate lateral and temporal resolution. At low pressure conditions, the first evidence of spatio-temporal pattern formation during the $\mathrm{CO}$ oxidation on Pt surfaces was observed with scanning low energy electron diffraction (LEED). ${ }^{16}$ However, the lateral and temporal resolution were not sufficient with $\sim 1 \mathrm{~mm}$ and several seconds, respectively. These findings encouraged the development of new techniques capable of imaging surface reactions. In early attempts to improve the resolution, an UV lamp was focused onto the sample to a spot size of less than $1 \mu \mathrm{m}^{2}$. The total yield of photoelectrons emitted from this small spot was collected by a channeltron. By scanning the surface in the focal plane in both the $x$ and $y$ directions, the scanning photoemission microscope (SPM) produced an image of the local work function over the investigated area. ${ }^{14,17,18}$ The next step in the evolution of surface imaging tools was the photoelectron emission microscope (PEEM). ${ }^{19}$ It was constructed as an "add on" microscope for any standard UHV chamber. The PEEM revealed an abundance of previously unobserved dynamic patterns in surface reactions. ${ }^{8}$ It has a spatial resolu- 


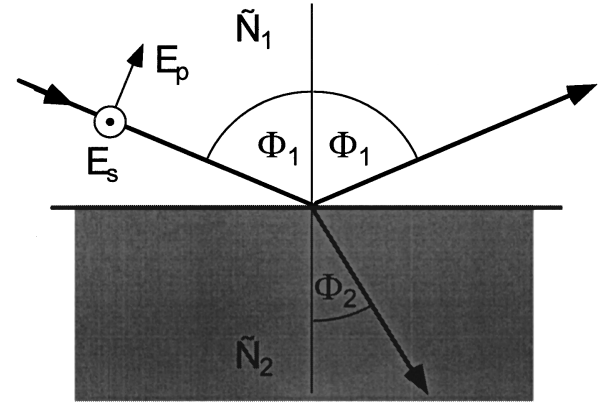

FIG. 1. Schematic drawing of the principle components of the electric field vector involved in a reflection experiment using plane polarized light.

tion of about $0.2 \mu \mathrm{m}$ and a temporal resolution of $20 \mathrm{~ms}$. One general limitation of the PEEM is that it can only operate under high vacuum conditions. Whenever electrons are used to carry information from a sample surface, experiments are only possible under vacuum conditions providing an appropriate mean free path for the electrons. Therefore, the PEEM is not capable of imaging surface reactions at pressures above $\sim 10^{-3}$ mbar.

The high surface sensitivity of optical methods, such as ellipsometry and reflection anisotropy spectroscopy, have been widely applied in surface studies. ${ }^{20,21}$ However, until recently, their submonolayer surface sensitivity has not been exploited to obtain spatial information in ultrathin adsorbate layers. The evolution of these methods to EMSI and RAM is a very promising step towards bridging the "pressure gap" between UHV investigations and studies at atmospheric pressures. ${ }^{1,2}$ Of these new techniques, EMSI will be the sole focus of this article and its underlying principles will now be discussed in detail.

\section{THEORY}

Ellipsometry is based on specular reflection of polarized light from surfaces. An advantage of using this imaging method is that the fundamental principles of the technique are well known. When light irradiates a surface, part of it is refracted and part of it is reflected. The part of the light beam that enters the material at an angle $\Phi_{1}$, is refracted to a different angle $\Phi_{2}$, as described by Snell's law:

$$
\widetilde{N}_{1} \sin \Phi_{1}=\widetilde{N}_{2} \sin \Phi_{2},
$$

where $\widetilde{N}_{i}$ is the complex index of refraction given as $n$ $-j k,(n$, index of refraction and $k$, extinction coefficient). $n$ and $k$ are both dependent on wavelength, therefore light of different wavelengths will be bent at different angles when incident on a refracting material.

In Fig. 1, the principal components involved in a reflection experiment using linear polarized light are shown. The angles of incidence, reflection, and refraction are all measured from the normal to the surface. Therefore, the incident light, upon reflection, defines the plane of incidence. The incoming linear polarized light on the surface can be split into a wave parallel to the plane of incidence, $E^{p}$, and one perpendicular to that plane, $E^{s}$. The ratio of the two (complex) electric field components $\hat{E}^{i}$ may be described as

$$
\frac{E^{p}}{E^{s}}=\tan (\varphi) e^{j \delta}
$$

where $\tan \varphi$ defines the amplitude ratio while $\delta$ characterizes the phase difference between the $s$ and $p$ components $(j$ $=\sqrt{-1}$ ). For $\delta=0$ or $\delta=\pi$ the light is linearly polarized, for $\delta=1 / 2 \pi$ or $\delta=3 / 2 \pi$ and $\tan \varphi=1$ or -1 it is circularly polarized, while all other cases constitute elliptically polarized light. At a certain angle, the $p$ component of reflected polarized light is minimized. This angle, called the Brewster or principal angle, $\Phi_{p}$, lies typically between $55^{\circ}$ and $80^{\circ}$ and depends on $n$ and $k_{\mathrm{i}}$, i.e., $\Phi_{p}$ is different for each substrate material. When both media are dielectric, e.g., glass in air, then $k=0$, and the $p$ component is zero. The $s$-polarized component of the light which is oriented perpendicular to the incident plane, increases steadily with $\Phi$.

Fresnel's equations define a reflection coefficient, $r$, as the ratio of the amplitude of the reflected wave to the amplitude of the incident wave for a single interface. These coefficients for perpendicular and parallel light are given by:

$$
\begin{array}{r}
r^{p}=\frac{\widetilde{N}_{2} \cos \Phi_{1}-\widetilde{N}_{1} \cos \Phi_{2}}{\widetilde{N}_{2} \cos \Phi_{1}+\widetilde{N}_{1} \cos \Phi_{2}}, \\
r^{s}=\frac{\widetilde{N}_{1} \cos \Phi_{1}-\widetilde{N}_{2} \cos \Phi_{2}}{\widetilde{N}_{1} \cos \Phi_{1}+\widetilde{N}_{2} \cos \Phi_{2}} .
\end{array}
$$

The reflectance, $R$, is defined as the ratio of reflected intensity to the incident intensity. Since the intensity of light is proportional to the square of the amplitude, this can be written as:

$$
R^{p}=\left|r^{p}\right|^{2} \quad \text { and } R^{s}=\left|r^{s}\right|^{2} .
$$

For ellipsometric measurements, the principal values to be determined are the phase change $\Delta$, and the ratio of incident to reflected amplitudes, $\psi$. The change in phase, $\Delta$, which occurs upon reflection is defined as:

$$
\Delta=\delta_{1}-\delta_{2}, \quad 0^{\circ}<\Delta<360^{\circ},
$$

where $\delta_{i}$ are the phase differences between the $p$ and $s$ components of the incoming and outgoing waves, respectively. The ratios $\left|R^{i}\right|$ of the outgoing wave amplitude to the incoming wave amplitude for the $p$ and the $s$ components define $\psi$ :

$$
\tan \psi=\frac{\left|R^{p}\right|}{\left|R^{s}\right|}, \quad 0^{\circ}<\psi<90^{\circ} .
$$

These equations are only applicable to single interfaces. However, many real world situations involve multiple interfaces, as shown in Fig. 2. Therefore, if we consider thin films (or adsorbates) on a surface, the above equations have to be expanded. The ratio of amplitude of the reflected wave to the amplitude of the incident wave is now given by the total reflection coefficient $R$ for the parallel and perpendicular components of light: 


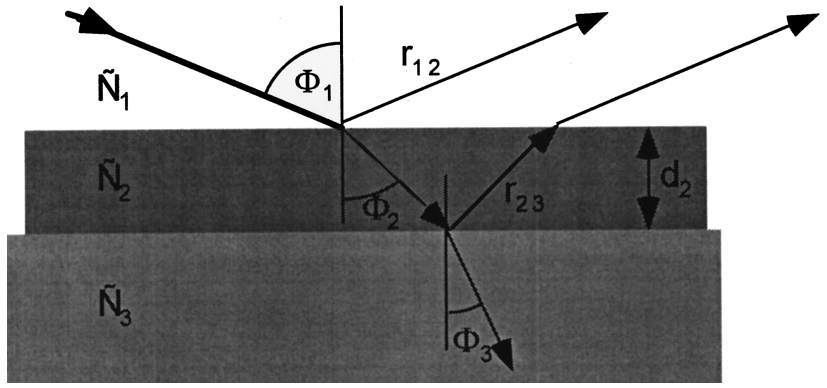

FIG. 2. Reflections and transmissions with multiple interfaces.

$$
\mathscr{B}_{13}^{p}=\frac{r_{12}^{p}+r_{23}^{p} e^{-i 2 \beta_{2}}}{1+r_{12}^{p} r_{23}^{p} e^{-i 2 \beta_{2}}}, \quad \mathscr{B}_{13}^{s}=\frac{r_{12}^{s}+r_{23}^{s} e^{-i 2 \beta_{2}}}{1+r_{12}^{s} r_{23}^{s} e^{-i 2 \beta_{2}}},
$$

where $\beta_{2}$ is given by:

$$
\beta_{2}=2 \pi \frac{d_{2}}{\lambda} \widetilde{N}_{2} \cos \Phi_{2},
$$

and $d_{2}$ is the film thickness. As a consequence, the equation for $\psi$ changes to:

$$
\tan \psi=\left|\frac{R^{p}}{R^{s}}\right| .
$$

The two quantities $\Delta$ and $\psi$ completely describe the ellipsometric experiment. This experiment is understood as the change in polarization upon reflection and is described by: $:^{20,21}$

$$
\rho=\frac{\mathscr{R}^{p}}{\mathscr{R}^{s}}=\tan \psi e^{j \Delta} .
$$

The quantities $\Delta$ and $\psi$ are measured using the ellipsometer. The calculated sample parameters, such as film thickness and index of refraction, depend on the correct model for the system under investigation. This can be very difficult at times.
Even unknown roughness of the substrate can render erroneous results if it has not been accounted for in the model. ${ }^{20}$

When measuring extremely thin films such as submonolayer coverages of adsorbates on a metal surface, the Drude approximation can be used. Both $\Delta$ and $\psi$ are assumed to change linearly with the thickness of the film:

$$
\begin{aligned}
& \Delta-\Delta_{c}=C_{\Delta} x \\
& \psi-\psi_{c}=C_{\psi} x
\end{aligned}
$$

where $\Delta_{c}$ and $\psi_{c}$ represent the values of the surface without the film, $x$ is the thickness of the film (i.e., the coverage), and $C_{\Delta}$ and $C_{\psi}$ are proportionality constants. Generally, changes in $\Delta$ are much more significant and changes in $\psi$ are neglected. The constant $C_{\Delta}$ can be calculated for a complete monolayer of adsorbate and then used when measuring fractions of a monolayer. The contrast in an EMSI image of a surface arises from local differences of the optical properties. For an adsorbate system, contrasts also arise from local variations of the coverages, $x$ and the change in intensity is mainly due to variations of $\Delta$. Therefore, the contrast can be directly calibrated into differences of the adsorbate coverage.

\section{EXPERIMENT}

The setup for EMSI is shown in Fig. 3. The instrument has the general outlines of a nulling ellipsometer, with the addition of an imaging lens, a CCD detector for obtaining spatial resolution, a "coherence scrambler," and a background subtraction unit for image enhancement. The light source consists of an argon ion laser at a wavelength of 514 $\mathrm{nm}$. A monochromatic source is necessary to achieve a high image contrast. Typically, a power of $200-400 \mathrm{~mW}$ is used in the experiments. For "coherence scrambling," the light is directed into a $20 \mathrm{~m}$ long multimode fiber optic light guide, several loops of which are vibrated by a loudspeaker. This is necessary because the high coherence length of laser light causes imperfections in the optical path, such as dust par-

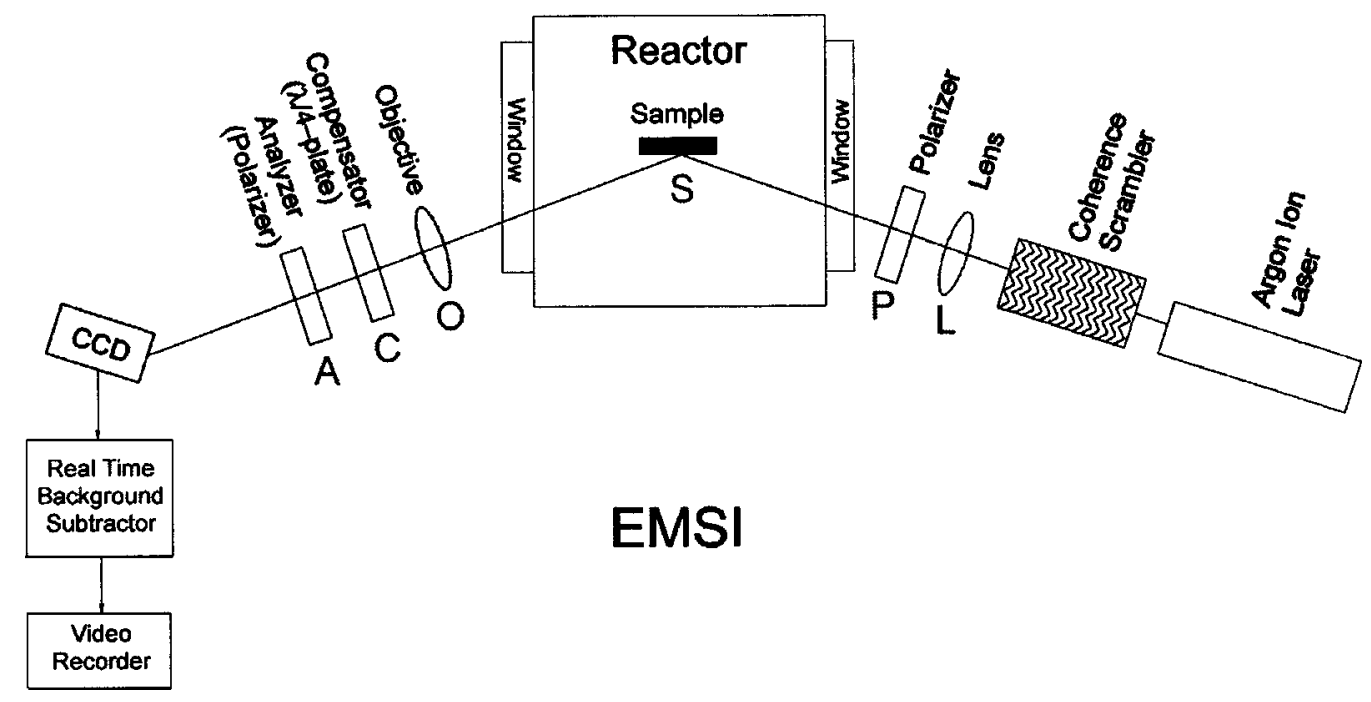

FIG. 3. Schematic diagram of the EMSI instrument setup. 
ticles, aberrations in the optical elements, or surface defects, to produce strong interference patterns in the final image. By vibrating a portion of the fiber optics, the different modes of beam propagation in the light guide are constantly changed reducing this problem. The light guide also causes an increase in the divergence of the light beam and a loss of intensity of approximately $40 \%$. Located at the end of the optical fiber is a condenser lens, $L, f=20 \mathrm{~mm}$. The beam from the lens passes through a Glan-Thompson polarizer, $P$, producing linearly polarized light that is directed onto the sample surface through a quartz window in the reactor. The light is specularly reflected off the sample surface, $S$, at an angle of approximately $70^{\circ}$ close to the Brewster angle. The reflected light exits the reactor through another quartz window and continues through an enlarger objective, $O, f=75$ $\mathrm{mm}$, which focuses an image of the sample onto the chip of a CCD camera. Real time background subtraction on the image is performed by use of an image processing unit (Hamamatsu Argus 20). Background subtraction is necessary to eliminate any residual interference patterns. The reactor windows in the setup have little effect on the performance of the instrument. This has been verified by operation at ambient conditions both with and without the windows in place. A zero-order quarter wave plate, $C$, and another GlanThompson polarizer, $A$, function as the compensator and analyzer, respectively. The compensator and analyzer are adjusted to produce a null or minimum intensity in the final image when the sample surface is in a homogeneous state. Usually at this stage, an image is stored and continually subtracted as a background to remove remaining interference patterns of the laser light. Since the polarization is sensitive to submonolayer adsorbate coverages, any change in the adsorbate layer will cause a change in the elliptical polarization of the light reflected from the surface. The change in the polarization state of the reflected light prevents it from now being extinguished by the compensator and analyzer which were adjusted to produce a null with the surface in a homogeneous state. Areas of varying intensity in the image therefore provide information about species on the sample surface. Usually, maximum contrast in the image is not achieved when the compensator and analyzer are adjusted such that the light reflected from one of the adsorbates is extinguished, but instead optimal operation lies somewhat apart from these settings.

Maximum contrast can be quantified by performing a series of measurements with the sample surface in a homogeneous state, i.e., completely $\mathrm{CO}$ covered or completely $\mathrm{O}$ covered. First, for an O-covered surface, the setting of maximum extinction is searched for with the compensator and analyzer, while the polarizer angle is kept fixed. By then leaving the compensator angle unchanged, the angle of the analyzer is varied and the overall intensity of the image is recorded, producing a parabolic increase in brightness as seen in Fig. 4. Using the same setting of polarizer and compensator, a similar curve is produced with the surface in a CO-covered state. From these curves, it is clear that a contrast exists to distinguish between $\mathrm{CO}$ and $\mathrm{O}$ covered areas

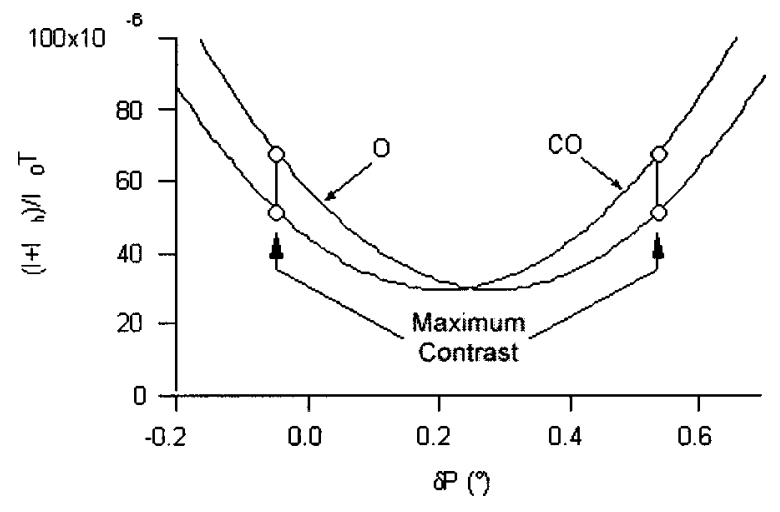

FIG. 4. Parabolic curves are produced when image intensity vs polarizer angle is measured for entirely covered oxygen and $\mathrm{CO}$ surfaces, respectively. The zero for the angle was set to give an optimal extinction for the clean surface.

on the surface. The maximum contrast is not achieved when the extinction is adjusted for one of the two states due to the large base signal. The base signal is a result of imperfections in the optical elements employed and the non-parallel incident light on the surface. The identification of the species on grounds of intensity alone is not possible, as two contrast conditions exist depending on the setting of the analyzer. Thus, O-covered areas may appear darker or lighter than $\mathrm{CO}$-covered areas depending on the conditions chosen.

Successful operation of the EMSI instrument requires that the optical elements are carefully aligned. It is important that the compensator, $C$, and analyzer, $A$, are finely adjusted. The intensity and parallelism of the incident beam are also factors. Usually, the repeated adjustment of each of these mentioned items is important to producing an adequate intensity contrast.

In Fig. $5,{ }^{22}$ an example of a typical EMSI image of pattern formation on a $\operatorname{Pt}(110)$ surface under high vacuum conditions is shown. ${ }^{23}$ The spatial resolution of the instrument is limited by diffraction. It has been determined to be approximately $4 \mu \mathrm{m}$ in the present setup. The temporal resolution is $30 \mathrm{~ms}$ given by the frequency of the video system with ample opportunity for improvement using faster video hardware. In a conventional imaging setup, with the camera plane perpendicular to the optical axis, the image of the sample would be strongly distorted and not sharp towards the edges due to the oblique imaging conditions. By tilting the CCD chip in the plane of incidence so that the angle of incidence

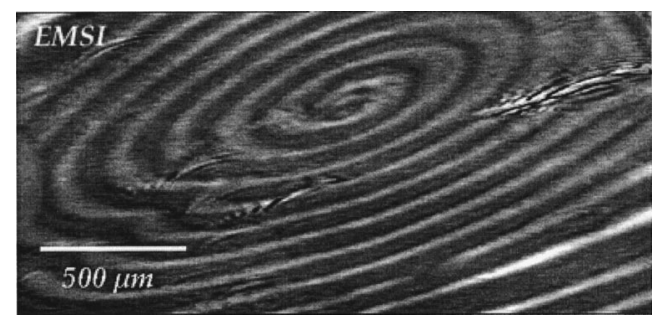

FIG. 5. EMSI image of a spiral wave pattern formed during $\mathrm{CO}$ oxidation on a $\mathrm{Pt}(110)$ surface. $P_{\mathrm{CO}}=7.8 \times 10^{-5}$ mbar, $P_{\mathrm{O}_{2}}=4 \times 10^{-4}$ mbar at $T=492 \mathrm{~K}$. 

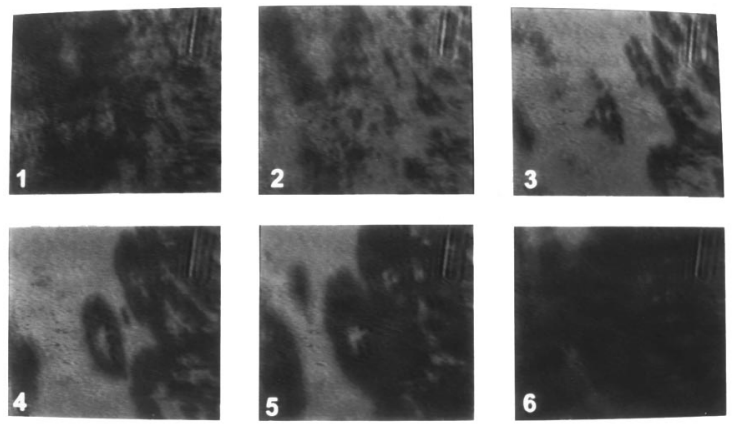

FIG. 6. Sequence of EMSI snapshots (image size $\sim 2.1 \mathrm{~mm} \times 2.5 \mathrm{~mm}$ ) showing pattern formation on the $\mathrm{Pt}(100)$ surface at 23 mTorr total pressure, $p_{\mathrm{CO}} / p_{\mathrm{O}_{2}}=0.08, T=523 \mathrm{~K}$. At these parameters, self-sustained rate oscillations are observed. The first two images ( $\Delta t=1 \mathrm{~s}$ between all frames) were taken during the transition from the oxygen covered to the $\mathrm{CO}$ covered state. Frames 3-6 then show the reverse transition into the high reactive state, which is now accompanied by the nucleation and growth of oxygen islands.

of the light onto the camera equals that at the sample, this effect is largely reduced and results in a nearly undistorted image.

\section{EXPERIMENTAL RESULTS}

As an example of the capability of EMSI to reveal new spatio-temporal phenomena at elevated pressures, we demonstrate that pattern formation plays an important role during the CO oxidation on $\mathrm{Pt}(100)$ surface. The first surface science study of an oscillatory reaction reported kinetic oscillations during $\mathrm{CO}$ oxidation on a $\mathrm{Pt}(100)$ single crystal. ${ }^{24}$ Oscillations were observed using a mass spectrometer monitoring the $\mathrm{CO}_{2}$ production rate and a Kelvin probe to measure work function differences. The time between the work function maxima varies on the order of minutes. A detailed analysis of spatio-temporal pattern formation at pressures of $10^{-4}$ mbar on $\mathrm{Pt}(100)$ was performed by Lauterbach and Rotermund using the PEEM. Only irregular rate oscillations were observed at these pressures, always coupled to pattern formation of the catalyst surface. ${ }^{25}$

Investigations of reaction fronts at higher partial pressures and temperatures were until recently severely restricted by the vacuum requirements of PEEM. The development of EMSI now allows the study of reaction fronts at any pressure. At much higher pressures of approximately 23 mTorr, compared to the previous study, ${ }^{25}$ the $\mathrm{CO}$ oxidation on $\operatorname{Pt}(100)$ exhibits regular, periodic self-sustained reaction rate oscillations. These oscillations are always related to the formation of dynamic patterns on the catalyst surface. A series of EMSI pictures is shown in Fig. 6. This sequence shows different surface states for one complete cycle of oscillation. The averaged $\mathrm{CO}_{2}$ signal, measured at the reactor outlet by means of a differentially pumped quadrupole mass spectrometer, proves the existence of self-sustained kinetic rate oscillations. These images show the pattern formation as the surface performs one oscillation cycle, where it transforms from the oxygen covered (dark areas) to the CO covered state (light gray) and then back to the oxygen covered state. The forward transition, frames 1 and 2, is characterized by the nucleation and growth of a large number of small $\mathrm{CO}$ islands. $\mathrm{CO}$ reaction diffusion fronts originating at these islands move over the surface until it is almost entirely $\mathrm{CO}$ covered. The reaction rate decreases during this process because the surface becomes more and more $\mathrm{CO}$ poisoned. Frames 3-6 show the reverse transition back to the highly reactive state, which is characterized by the nucleation and growth of oxygen islands on the otherwise $\mathrm{CO}$ poisoned surface. Gas-phase oxygen needs two free adjacent adsorption sites and can only penetrate the $\mathrm{CO}$ adlayer at surface defects. From these nucleation centers, oxygen reaction diffusion fronts then transform the surface from the $\mathrm{CO}$ covered to the oxygen covered state. ${ }^{25}$ Also note the difference in the size of the $\mathrm{CO}$ fronts versus the oxygen fronts.

\section{CONCLUSIONS}

EMSI has been developed out of our interest in nonlinear phenomena and spatio-temporal self organization in heterogeneous catalysis. It allows the imaging of concentration patterns in the adsorbate layer on catalyst surfaces at arbitrary pressures. Its unique sensitivity to image the distribution of ultrathin films and submonolayer coverages of adsorbates under arbitrary external conditions make it a valuable tool for a wide variety of investigations.

${ }^{1}$ H. H. Rotermund, G. Haas, R. U. Franz, R. M. Tromp, and G. Ertl, Science 270, 608 (1995).

${ }^{2}$ H. H. Rotermund, G. Haas, R. U. Franz, R. M. Tromp, and G. Ertl, Appl. Phys. A: Solids Surf. 61, 569 (1995).

${ }^{3}$ H. Beusch, P. Fieguth, and E. Wicke, Chem. Ing. Tech. 44, 445 (1972).

${ }^{4}$ M. Sheintuch and R. A. Schmitz, Catal. Rev. Sci. Eng. 15, 107 (1977).

${ }^{5}$ F. Schuth, B. E. Henry, and L. D. Schmidt, Adv. Catal. 39, 51 (1993).

${ }^{6} \mathrm{G}$. Ertl, in Advances in Catalysis (Academic, New York, 1990), Vol. 37, p. 213.

${ }^{7}$ G. Ertl, Surf. Sci. 287, 1 (1993).

${ }^{8}$ G. Ertl and H. Rotermund, Solid State Mater. Sci. 1, 617 (1996).

${ }^{9}$ R. Imbihl, Prog. Surf. Sci. 44, 185 (1993).

${ }^{10}$ R. Imbihl, Heterog. Chem. Rev. 1, 125 (1994).

${ }^{11}$ R. Imbihl and G. Ertl, Chem. Rev. 95, 697 (1995).

${ }^{12}$ M. Eiswirth, M. Bär, and H. H. Rotermund, Physica D 84, 40 (1995).

${ }^{13}$ S. Jakubith, H. H. Rotermund, W. Engel, A. von Oertzen, and G. Ertl, Phys. Rev. Lett. 65, 3013 (1990).

${ }^{14} \mathrm{H}$. H. Rotermund, S. Jakubith, A. Von Oertzen, and G. Ertl, J. Chem. Phys. 91, 4942 (1989).

${ }^{15}$ H. H. Rotermund, Phys. Scr. T49B, 549 (1993).

${ }^{16}$ M. P. Cox, G. Ertl, and R. Imbihl, Phys. Rev. Lett. 54, 1725 (1985).

${ }^{17}$ H. H. Rotermund, G. Ertl, and W. Sesselmann, Surf. Sci. 217, L383 (1989).

${ }^{18}$ H. H. Rotermund, S. Jakubith, S. Kubala, A. Von Oertzen, and G. Ertl, J. Electron Spectrosc. Relat. Phenom. 52, 811 (1990).

${ }^{19}$ H. H. Rotermund, W. Engel, S. Jakubith, A. Von Oertzen, and G. Ertl, Ultramicroscopy 36, 164 (1991).

${ }^{20} \mathrm{H}$. G. Tompkins, A User's Guide to Ellipsometry (Academic, Boston, 1993).

${ }^{21}$ R. M. A. Azzam and N. M. Bashara, Ellipsometry and Polarized Light (North-Holland, New York, 1977).

${ }^{22}$ G. Haas, Dissertation, Freie Universität Berlin, Germany, 1997.

${ }^{23}$ S. Nettesheim, A. Von Oertzen, H. H. Rotermund, and G. Ertl, J. Chem. Phys. 98, 9977 (1993).

${ }^{24}$ G. Ertl, P. R. Norton, and J. Rüstig, Phys. Rev. Lett. 49, 177 (1982).

${ }^{25}$ J. Lauterbach and H. H. Rotermund, Surf. Sci. 311, 231 (1994). 